\title{
Eficacia de la utilización de estilos de aprendizaje en conjunto con mapas conceptuales y aprendizaje basado en la resolución de problemas para el aprendizaje de neuroanatomía
}

J.O. Ayala-Pimentel, J.A. Díaz-Pérez, L.C. Orozco-Vargas

Introducción. La utilización combinada de estilos de aprendizaje en conjunto con mapas conceptuales y el empleo de aprendizaje basado en la resolución de problemas (EMCRP) es una nueva estrategia educativa. Objetivo. Evaluar la eficacia de la utilización del método EMCRP en la adquisición de aprendizaje significativo de neuroanatomía, comparado con el método usual de aprendizaje en estudiantes de fisioterapia, que cursaron la asignatura morfofisiología general en la Universidad Industrial de Santander (UIS) entre los años 2004 y 2007. Sujetos y métodos. Se utilizó un diseño experimental con participantes aleatorizados asignados a dos grupos con una relación 1 a 1. En el grupo intervenido se empleó el método EMCRP y en el control el método tradicional de enseñanza. Después de un año se evaluó la adquisición de aprendizaje significativo para determinar el rendimiento del método EMCRP. Resultados. Se estudiaron 55 estudiantes. La edad media fue de 23 años y la razón mujer-hombre fue de 3 a 1. Al evaluar a los estudiantes después de un año de la intervención, 15 del grupo intervenido reprobaron el examen frente a 26 del grupo control (55 frente a 92\%; $p=0,002$ ). Se determinó una reducción del riesgo absoluto de 0,37 (intervalo de confianza al 95\% = 0,16-0,56) y número necesario para tratar de 2,7 (intervalo de confianza al 95\% = 1,7-6,3). Conclusión. La adquisición de un aprendizaje significativo fue mayor en el grupo intervenido, evidenciado por una menor proporción de suspendidos importante en comparación con el grupo control, con un número de estudiantes bajo a intervenir para que se produzcan resultados favorables.

Palabras clave. Aprendizaje basado en problemas. Ensayo experimental. Morfología. Neuroanatomía.

\section{Effectiveness of using learning styles in conjunction with conceptual maps based learning and problem solving for teaching neuroanatomy}

Introduction. The use of learning styles combined with concept maps and the use of learning based on the resolution of problems (LSCMLRP) are a new educational strategy. Aim. To evaluate the effectiveness of the method LSCMLRP in the acquisition of significantlearning of neuroanatomy, compared with the usual method of learning in physiotherapy students. These students, attended the subject general morphofisiology in the Industrial University of Santander (UIS) between the years 2004 and 2007. Subjects and methods. A controlled clinical test was carried out to determine the performance of the LSCMLRP. The students were randomized and assigned to two groups with a 1:1 relation. In the treated group the LSCMLRP method was used and in the control the lecture method. Subsequent to a period of 1 year the acquisition of significant learning was evaluated. Results. 55 students were studied. The average age was of 23 years and the ratio woman: man was of 3:1. When evaluating the students after 1 year of the intervention 15 students of the treated group failed the examination compared to 26 students of the control group control ( $55 \%$ versus $92 \%, p=0.002$ ). The calculated absolute risk reduction of $0.37(\mathrm{Cl} 95 \%=0.16-0.56)$ and NNT 2,7 (C) $95 \%=1.7-6.3)$. Conclusion. The acquisition of significant learning was greater in the treated group, demonstrated by a significant minor proportion of exam failures compared to the control group, and with a low student to intervene in order to produce favorable outcomes.

Key words. Morphology. Neuroanatomy. Problem-based learning. Randomized controlled trial.
Grupo de Investigación en Estrategias para la Enseñanza en Ciencias de la Salud. Universidad Industrial de Santander. Bucaramanga, Colombia.

Correspondencia Dr. Jaime O. Ayala Pimentel. Departamento de Ciencias Básicas. Unidad de Neuroanatomía. Facultad de Salud. Universidad Industrial de Santander. Cra. 32, n. 29-31. Bucaramanga, Colombia.

E-mail jayala@uis.edu.co 


\section{Introducción}

Las ciencias que estudian el sistema nervioso son consideradas por muchos estudiantes como de difícil comprensión $[1,2]$, por lo cual escogen como única opción para aprobarlas memorizar la mayor cantidad posible de información [1-4]. A este temor se suma una temática extensa, con una amplia cantidad de conceptos complejos y la tendencia, por parte de los docentes, a utilizar modelos donde no existe la participación activa del estudiante [1,5]. En la Universidad Industrial de Santander (UIS), Colombia, en el caso de la carrera de fisioterapia, la anatomía del sistema nervioso forma parte de una asignatura denominada morfofisiología general. Los métodos de enseñanza empleados regularmente para su desarrollo son la clase magistral, la disección [6] y el estudio de estructuras anatómicas en especímenes cadavéricos [7]. A pesar de estos múltiples métodos de enseñanza, existe una alta tasa de suspendidos, por lo cual fue diseñada una nueva metodología que incluye la utilización de estilos de aprendizaje en conjunto con mapas conceptuales y aprendizaje basado en la resolución de problemas (EMCRP), los cuales otorgan un valor importante al autoaprendizaje y a la evaluación formativa, cualitativa e individualizada $[8,9]$. La pretensión de este enfoque pedagógico, multimetodológico y multididáctico es enseñar y estimular al alumno a aprender mediante la formación de grupos de estudiantes con un mismo estilo de aprendizaje [10], que con la mediación de un tutor analizan y resuelven un interrogante seleccionado o diseñado especialmente para el logro de ciertos objetivos de aprendizaje. Durante este proceso de interacción, los alumnos resuelven un problema o construyen un mapa conceptual, con lo cual se pretende lograr, además del aprendizaje del conocimiento propio de la materia, que elaboren un diagnóstico de sus propias necesidades de aprendizaje, que comprendan la importancia de trabajar de forma colaborativa, que desarrollen habilidades de análisis y que sinteticen información; además de comprometerse con su proceso de aprendizaje, todo esto de acuerdo con las técnicas didácticas propias de su estilo de aprendizaje [11-15]. Este nuevo método de enseñanza de la neuroanatomía no ha sido probado, por tanto se traza como objetivo evaluar la eficacia de la utilización del método EMCRP en la adquisición de aprendi- zaje significativo de neuroanatomía, comparado con el método usual de aprendizaje en estudiantes de fisioterapia en la UIS.

\section{Sujetos y métodos}

\section{Diseño del estudio}

Se utilizó un diseño experimental entre julio de 2004 y septiembre de 2007, con el fin de evaluar el aprendizaje logrado con el método EMCRP. En el segundo semestre de 2004 se intervino un grupo de estudiantes con la metodología que se debía evaluar y se comparó con un segundo grupo que recibió la enseñanza habitual, con igual intensidad horaria teórica y práctica y nivel formativo de los docentes (médicos con maestría en morfología y especialización en docencia universitaria), e igualdad de horarios, durante el primer semestre de 2006. Este estudio fue diseñado, evaluado y analizado por el mismo grupo de investigadores. El proceso de selección de los grupos, análisis y evaluación se realizó de forma enmascarada con respecto al método de enseñanza utilizado y otros datos de identificación.

\section{Estudiantes}

La población que se estudió fueron los alumnos de primer año de fisioterapia, quienes cursaban la materia de morfofisiología general, en el área de neuroanatomía. Se excluyeron los que cursaban de nuevo la asignatura. Los alumnos que formaron parte de este estudio fueron asignados de forma enmascarada y aleatoria a través de una tabla de números al azar a dos grupos con una relación de 1 a 1 . El grupo de intervención estuvo formado por 27 estudiantes y el grupo control por 28. Después de cursar la asignatura, se hizo un seguimiento de los grupos durante un año y se evaluaron con la misma herramienta, previamente diseñada para este objetivo, que calificaba de forma cuantitativa el rendimiento del aprendizaje logrado (Figura).

\section{Grupo experimental}

En este estudio se utilizó el método EMCRP, que consiste en la caracterización de los estilos de aprendizaje de los estudiantes y la aplicación de una metodología durante 20 horas desarrolladas 


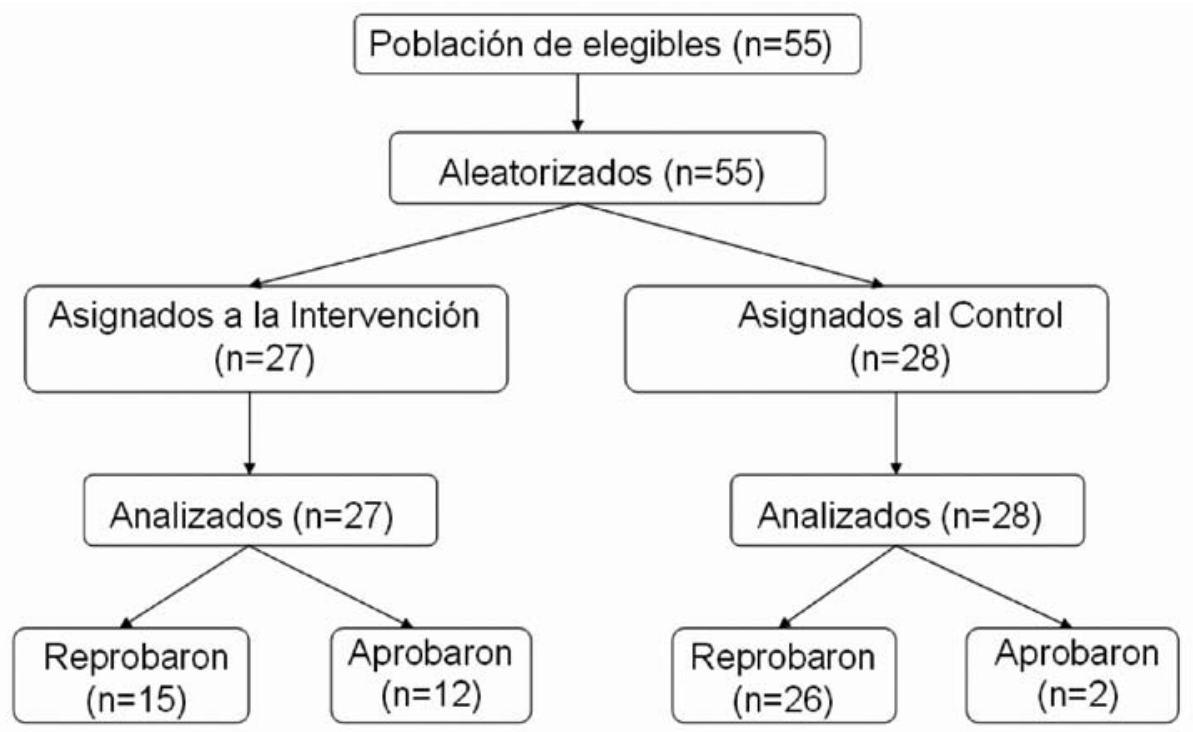

Figura. Diagrama de flujo de los estudiantes a través de las fases del experimento.

en un período de 6 semanas, que constaba de los tres componentes que se citan a continuación.

\section{Primera etapa: caracterización} de los estilos de aprendizaje

El primer día de clase los estudiantes desarrollaron el cuestionario 'Diagnóstico de estilos de aprendizaje' [16], cuyos resultados permitieron la formación de grupos de trabajo de tres a cinco estudiantes de un mismo estilo de aprendizaje, a partir de los cuales se trabajaron las 20 horas de clase teórica programadas para el semestre, con un único profesor para todos los grupos.

\section{Segunda etapa: explicación de}

la metodología de trabajo en clase

Al inicio de cada sesión se explicaba a los estudiantes los objetivos de aprendizaje y la dinámica que se desarrollaría. Ésta consistía en la utilización de una de las estrategias siguientes: elaboración o análisis de mapas conceptuales, lectura y análisis de casos clínicos y formulación de secuencias neurofuncionales basadas en situaciones de la vida cotidiana, por ejemplo, la diagramación del circuito neuroanatómico utilizado en la sensación de dolor después de una quemadura.

Los estudiantes debían utilizar una de estas situaciones-problema para conocer, relacionar y comprender las estructuras neuroanatómicas y sus circuitos. Ya en grupo, los estudiantes realizaban la lectura crítica del material entregado, se distribuían los roles de trabajo y planeaban el proceso que debían seguir. Para clarificar, analizar y solucionar la tarea asignada por el profesor a los estudiantes, los cuales debían consultar diferentes fuentes bibliográficas. Al finalizar la jornada, los grupos hacían dos copias del resultado de su trabajo; una para entregar al profesor y otra para realizar la corrección y autoevaluación de su trabajo.

Tercera etapa: trabajo independiente

El estudiante analizaba el trabajo realizado en clase, consultaba nueva bibliografía y complementaba el documento desarrollado.

\section{Cuarto etapa: socialización}

de análisis y resultados

En sesión plenaria, cada grupo presentaba los resultados de su trabajo, respondían preguntas y clarificaban ideas, de manera que enriquecían sus puntos de vista con las aportaciones de los otros grupos. El profesor completaba la explicación y ayudaba a elaborar una solución mejorada e inducía a la reflexión sobre el proceso seguido. 
Tabla I. Características de los estudiantes participantes.

\begin{tabular}{lccc}
\hline & Grupo de intervención & Grupo control & $p$ \\
\hline Sexo (mujer:hombre) & $3: 1$ & $3: 1$ & 1 \\
\hline Edad (años) & 23 & 23,2 & 0,82 \\
\hline Estrato social & 3 & 3 & 1 \\
\hline Total & 27 & 28 & 1 \\
\hline
\end{tabular}

Además de las horas de trabajo teórico, los estudiantes asistían cuatro horas por semana al anfiteatro durante las seis semanas que duraba el módulo de estudio del sistema nervioso.

\section{Grupo de comparación}

La metodología utilizada como control se basó en la forma usual de enseñanza, la cual consistía en clases magistrales, en las que el profesor mediante ayudas audiovisuales explicaba el tema que debía desarrollar, con una intensidad de 20 horas durante seis semanas. El tiempo de trabajo en el anfiteatro fue de cuatro horas semanales durante las semanas que duró el módulo de neuroanatomía, al igual que el grupo de intervención.

\section{Evaluación de la eficacia}

En la evaluación de la eficacia de la adquisición de aprendizaje fue utilizado un examen de 25 preguntas de selección múltiple, de respuesta única, con cinco opciones, en el cual se evaluaba embriología, terminología, clasificación y morfología de la neuroanatomía básica; previamente, este examen lo revisaron expertos en la materia. Para aprobarlo, los estudiantes debían contestar correctamente 15 preguntas.

\section{Consideraciones éticas}

La presente investigación fue realizada acorde con la legislación actualmente vigente nacional (Constitución política nacional de Colombia de 1991 y el Decreto 008439 de 1993) e internacional (Declaración de Helsinki). Esta investigación recibió la aprobación de las directivas del curso de morfofisiología y los estudiantes fueron provistos de un consentimiento informado, utilizando un formato con las características propuestas por la legislación nacional. Se garantizó la confidencialidad de los datos recogidos.

\section{Análisis}

Para la realización de este estudio se calculó un tamaño de muestra para tener un poder del 0,8 y una diferencia entre los dos grupos del $50 \%$, con nivel de error $\alpha$ de 0,05 , el cual fue de 20 participantes en cada grupo. Por medio de un análisis estadístico se determinó la probabilidad de no aprobación del examen por parte del estudiante del grupo intervenido y del grupo control, y se calcularon sus diferencias por medio del test estadístico de chi al cuadrado. Además, se determinó la reducción del riesgo absoluto (RRA) y el número necesario para tratar o intervenir (NNT) al utilizar la metodología EMCRP. Se hallaron los intervalos de confianza al 95\% utilizando el método de regresión binomial. El análisis estadístico se realizó en el programa Stata 10.0 sobre una base de datos previamente elaborada en Excel 2003.

\section{Resultados}

\section{Población estudiada}

Las características de los estudiantes que participaron en este estudio se muestran en la tabla I y sus estilos de aprendizaje en la tabla II. 
Tabla II. Estilos de aprendizaje de los estudiantes de fisioterapia intervenidos con el método EMCRP.

\begin{tabular}{lc}
\hline Reflexivo & $12(48,1 \%)$ \\
\hline Pragmático & $6(22,2 \%)$ \\
\hline Teórico & $5(18,5 \%)$ \\
\hline Activo & $4(14,8 \%)$ \\
\hline
\end{tabular}

EMCRP: estilos de aprendizaje en conjunto con mapas conceptuales y el empleo de aprendizaje basado en la resolución de problemas

Tabla III. Eficacia de la evaluación del método EMCRP en estudiantes de neuroanatomía de la Universidad Industrial de Santander.

\begin{tabular}{ll} 
RRA & $0,37($ IC 95\% $=0,16-0,56)$ \\
\hline NNT & $2,70($ IC $95 \%=1,7-6,3)$
\end{tabular}

EMCRP: estilos de aprendizaje en conjunto con mapas conceptuales y el empleo de aprendizaje basado en la resolución de problemas; IC: intervalo de confianza; NNT: número necesario para tratar; RRA: reducción del riesgo absoluto.

\section{Eficacia}

En el grupo de intervención, 15 estudiantes de 27 (55\%) suspendieron el examen frente a 26 estudiantes de 28 (92\%) que formaban el grupo control, con una $p$ de 0,002 . En la tabla III se muestran los valores de eficacia en la adquisición de nuevas competencias con el método EMCRP.

\section{Discusión}

En la educación superior, la metodología utilizada tradicionalmente en la docencia universitaria ha sido magistral, teniendo en cuenta que este concepto no sólo describe el tipo de actividad docente, sino también una manera de entender el aprendizaje como un proceso en el cual la formación del estudiante pasa por proporcionarle unos conocimientos que ha de asimilar [17]. Dentro de este contexto, los procesos de innovación que consisten en la incorporación de nuevos materiales, la utilización de estrategias de enseñanza implicativas y constructivistas, y prácticas innovadoras por parte de los docentes, han permitido la obtención de mejores resultados en los procesos de enseñanza y aprendizaje $[15,18]$. Estos cambios hacen que estudiantes y docentes asuman roles diferentes a los de la figura clásica tradicional. Así pues, el profesor debe pasar de ser la fuente de información a ser el facilitador y mediador de procesos de aprendizaje, y el estudiante debe pasar de la pasividad a un compromiso permanente y responsable con su aprendizaje [19].

A partir de la premisa que el estudiante sea el protagonista de su propio aprendizaje e integre el conocimiento, se creó y aplicó un nuevo proceso didáctico de construcción de conocimiento basado en las teorías cognitivas de aprendizaje de Vygotsky y Pozo [20] y, principalmente, del aprendizaje significativo de David Ausubel [21]. Con esta propuesta educativa innovadora se buscaba, en un nuevo ambiente, convertir la enseñanza en un proceso dinámico y desarrollar la investigación en comunidad. Al adoptar parte de esta metodología en este trabajo, el grupo experimental fue formado por diferentes subgrupos de acuerdo con los resultados del cuestionario de estilos de aprendizaje de Honey-Alonso (CHAEA) $[10,16]$. Esto se basa en que el conocimiento del estilo de aprendizaje ofrece al estudiante sugerencias y aplicaciones prácticas con grandes posibilidades de conseguir un aprendizaje más efectivo de cara a un mejor rendimiento académico [22-27]. En el caso particular de este trabajo, se encontró que los estudiantes de fisioterapia obtenían mejor rendimiento cuando conocían su estilo de aprendizaje.

Los estudiantes utilizaron como estrategias pedagógicas los mapas conceptuales, los cuales permiten la organización jerárquica de los conceptos fundamentales que se han de aprender y la constatación de las relaciones que se dan entre ellos, y las relaciones de los conceptos previos con la nueva información. Su elaboración fomenta el conocimiento, el pensamiento reflexivo, la creatividad y el análisis crítico sobre un tema; así es cómo el mapa conceptual es, en consecuencia, un instrumento útil para la organización y la representación visual del conocimiento, puede 
ser elaborado por el docente para mostrar cómo se relacionan determinados conceptos o por el alumno, de forma individual o en grupo, con el objeto de alcanzar una mayor comprensión de los conceptos que estudia [28-30].

La utilización de situaciones-problema como casos clínicos, que son comunes en las diferentes facultades de ciencias de la salud para desarrollar tópicos distintos de cada una de las asignaturas [29,31-33]; además de la formulación de secuencias neurofuncionales basadas en situaciones de la vida cotidiana, estrategia desarrollada en la UIS, donde se utiliza una situación de la vida real para llegar a describir el recorrido de la información desde el momento en que es captada y analizada hasta cuando se genera una respuesta por parte del organismo. Con esta estrategia se consigue un mejor aprendizaje de las estructuras nerviosas y sus conexiones.

Este método permite una disminución estadísticamente significativa de la de perdida del examen la población intervenida comparada con la no intervenida del $40 \%$. Se muestra un NNT para tener un resultado favorable bajo (tres estudiantes) que avala el rendimiento de este método.

Adicionalmente, con la implementación de este método, que es compatible con la arquitectura cognitiva humana $[34,35]$, se puede favorecer en el estudiante el desarrollo de operaciones intelectuales y funciones cognitivas que mejoren su pensamiento científico por medio de las estrategias utilizadas, ya que se añade valor a los procesos de construcción del conocimiento de neuroanatomía, expresados en un cambio de actitud, de quien aprende de forma significativa. En este proceso, el propio estudiante construyó su conocimiento, y mostró interés y decisión por aprender.

Finalmente, podemos concluir que el conocimiento de los estilos de aprendizaje y el uso de una metodología constructivista permitió un mejor estudio de la neuroanatomía en los estudiantes de segundo semestre de fisioterapia. El enfoque metodológico utilizado se fundamentó en la construcción comunitaria del conocimiento mediante la interacción con las realidades donde se aplica, lo que permitió a los estudiantes ampliar su conocimiento.

\section{Bibliografía}

1. González-Álvarez JE. Enseñanza de la neurología en el pregrado: propuesta de una nueva metodología. Rev Chil Neuropsiquiatr 2004; 42: 131-7.

2. Jozelowicz R. Neurofobia: the fear of neurology among medical students. Arch Neurol 1994; 51: 328-9.

3. Purdy RA, Benstead TJ, Colmes DB, Kaufman DM. Using problem based learning in neurosciences education for medical students. Can J Neurol Sci 1999; 26: 211-6.

4. Ayala-Pimentel JO. Experiencia con el uso de mapas conceptuales en neuroanatomía. Revista Salud UIS 1999; 30: 65-7.

5. Diamond MC, Scheibel AB, Elson LM. The human brain coloring book. 1 ed. Oakville: Collins; 1986.

6. Montemayor-Flores B. El significado de la práctica de disección para los estudiantes de medicina. Int J Morphol 2006; 24: 575-80.

7. Winkelmann A. Anatomical dissection as a teaching method in medical school: a review of the evidence. Med Educ 2007; 41: 15-22.

8. Rodríguez SJ. Educación médica. Aprendizaje basado en problemas. México: Panamericana; 2002.

9. Rendas AB, Fonseca M, Rosado-Pintos P. Toward meaningful learning in undergraduate medical education using concept maps in PBL pathophysiology course. Adv Physiol Educ 2006; 30: 23-9.

10. Alonso CM, Gallego DJ, Honey P. Los estilos de aprendizaje: procedimientos de diagnóstico y mejora. Bilbao: Mensajero; 1994.

11. Ontoria A. Los mapas, otra forma de aprender. Revista Internacional Magisterio 2005-2006; 18: 22-6.

12. Ontoria A. Mapas conceptuales, una técnica para aprender. Madrid: Narcea; 1994.

13. Novack JD, Gowin DB. Aprendiendo a aprender. Barcelona: Martínez Roca; 1988.

14. Dueñas VH. El aprendizaje basado en problemas como enfoque pedagógico en la educación en salud. Colombia Médica 2001; 32: 189-96.

15. Hsu LL. Developing concept maps from problem-based learning scenario discussions. J Adv Nurs 2004; 48: 510-8.

16. Alonso C, Gallego D, Honey P. Cuestionario Honey-Alonso de estilos de aprendizaje CHAEA. URL: www.aprender. org.ar/aulas/avadim/recursos/chaea1.rtf [01.12.2008].

17. Corredor M, Arbeláez R, Pérez Ml. Estrategias de enseñanza y aprendizaje. Bucaramanga: UIS; 2007.

18. Michael J. In pursuit of meaningful learning. Adv Physiol Educ 2001; 25: 145-58. 
19. Díaz-Barriga F, Hernández G. Estrategias docentes para un aprendizaje significativo. Una interpretación constructivista. México: McGraw-Hill; 1999.

20. Pozo JI. Teorías cognitivas del aprendizaje. 8 ed. Madrid: Morata; 2003.

21. Ausubel D, Novak J, Hanesian H. Psicología educativa: un punto de vista cognoscitivo. 2 ed. México: Trillas; 1983.

22. Felder RM. Reaching the second tier: learning and teaching styles in college science education. J Coll Sci Teach 1993; 22: 286-90.

23. Lynch T, Wowlfl N, Steele D, Hanssen D. Learning style influences student examination performance. Am J Surg 1998; 176: 62-6.

24. Noble KA, Miller SM, Heckman J. The cognitive style of nursing students: educational implications for teaching and learning. J Nurs Educ 2008; 47: 245-53.

25. Bitran M, Lafuente M, Zúñiga D, Viviani P, Mena B. The influence of psychological features and learning styles on the academic performance of medical students. Rev Med Chil 2004; 132: 1127-36.

26. Tharp G. Relationship between personality type and achievement in an undergraduate physiology course. Am J Physiol 1992; 262: S1-3.

27. Boyle EA, Duffy T, Dunleavy K. Learning styles and academic outcome: the validity and utility of Vermunt's Inventory of Learning Styles in a British higher education setting. Br J Educ Psychol 2003; 73: 267-90.

28. Gallego-Badillo R, Pérez R. Corrientes constructivistas.
De los mapas conceptuales a la teoría de la transformación intelectual. Bogotá: Magisterio; 1997.

29. Johnstone $\mathrm{AH}$, Otis $\mathrm{KH}$. Concept mapping in problem based learning: a cautionary tale. Chemistry Education Research and Practice 2006; 7: 84-95.

30. Álvarez-Montero S, Caballero-Martin MA, Gallego-Casado $\mathrm{P}$, et al. Sesiones basadas en escenarios clínicos: una aplicación del aprendizaje basado en la resolución de problemas. Revista de Medicina Familiar y Comunitaria 2001; 11: 73-87.

31. Williams B. Case based learning -a review of the literature: is there scope for this educational paradigm in prehospital education? Emerg Med J 2005; 22: 577-81.

32. Krueger P, Neutens J, Bienstock J, Cox S, Erickson S, Goepfert A, et al. To the point: reviews in medical education teaching techniques. Am J Obstet Gynecol 2004; 191: 408-11.

33. Canalejas M, Martínez M, Pineda M, Vera M, Soto M, Martín A, et al. Estilos de aprendizaje en los estudiantes de enfermería. Educ Med 2005; 8: 33-40.

34. Kirschner PA, Sweller J, Clark R. Why minimal guidance during instruction does not work: an analysis of the failure of constructivist, discovery, problem based, experimental, and inquiry based teaching. Educational Psychologist 2006; 41: 75-86.

35. Schmidt HG, Loyens SMM, Van Gog T, Paas F. Problembased learning is compatible with human cognitive architecture. Educational Psychologist 2007; 42: 91-7. 